\title{
Intelligent Design for Neonatal Monitoring with Wearable Sensors
}

\author{
Wei Chen ${ }^{1}$, Sibrecht Bouwstra ${ }^{1}$, Sidarto Bambang Oetomo ${ }^{1,2}$ and Loe Feijs ${ }^{1}$ \\ ${ }^{1}$ Department of Industrial Design, Eindhoven University of Technology, \\ 2Department of Neonatology, Máxima Medical Center, Veldhoven, \\ The Netherlands
}

\section{Introduction}

Neonatal monitoring refers to the monitoring of vital physiological parameters of premature infants, full term infants that are critically ill, and a combination thereof. Babies that are born after a pregnancy lasting 37 weeks or less are typically considered premature. Critically ill neonates are a special group of patients that consist of premature infants who may suffer from diseases that are mainly caused by immaturity of their organs, and full term infants, who become severely ill during or immediately after birth. In particular, these premature infants can weigh as little as $500 \mathrm{~g}$ with a size of a palm and are highly vulnerable to external disturbances. Critically ill newborn infants are normally admitted to a Neonatal Intensive Care Unit (NICU) for treatment by neonatologists and specialized nurses.

Continuous health monitoring for the neonates provides crucial parameters for early detection of in adverted events (such as cessation of breathing, heart rhythm disturbances and drop in blood oxygen saturation), and possible complications (such as seizures). Immediate action based on this detection increases survival rates and positively supports further development of the neonates. Advances in medical treatments over the last decades resulted in a significant increase of survival. As a result, neonates born after 25 weeks of pregnancy can survive with adequate medical care and appropriate medical care in NICU (Costeloe et al., 2000). Encouraged by this success NICUs are populated by a large proportion of infants, born after very short gestational age. Survival and long-term health prospects strongly depend on medical care and reliable and comfortable health-status monitoring systems.

In the last decades several important treatment modalities emerged that had a substantial impact on the mortality of prematurely born infants. However there is a concomitant increase of neurobehavioral problems on long-term follow-up (Perlman, 2001; Hack \& Fanaroff, 1999; Chapieski \& Evankovitch, 1997). Follow-up studies indicate that preterm infants show more developmental delay compared to their full-term peers. More than $50 \%$ of them show deficits in their further development, such as visual-motor integration problems, motor impairments, speech and language delay, behavioral, attention, and learning problems (Marlow et al. 2007). Medical conditions including chronic lung disease, apnea and bradycardia, transient thyroid dysfunction, jaundice and nutritional deficiencies, are potential contributing factors. In addition infants in a busy NICU are often exposed to stressful environmental conditions. Examples are the attachment to multiple monitoring 
devices and intravenous lines, high noise levels and bright light (Perlman, 2003). A concept of interactions in the developing neonatal brain with maternal separation and exposure to pain and stress is illustrated in Fig. 1, according to Anand and Scalzo (Anand \& Scalzo, 2000). These negative stimuli can interfere with the normal growth and development of the neonates and hamper the parent-child interaction (Als et al., 2003). Thus, it is essential to develop comfortable care solutions for NICU and follow-up.
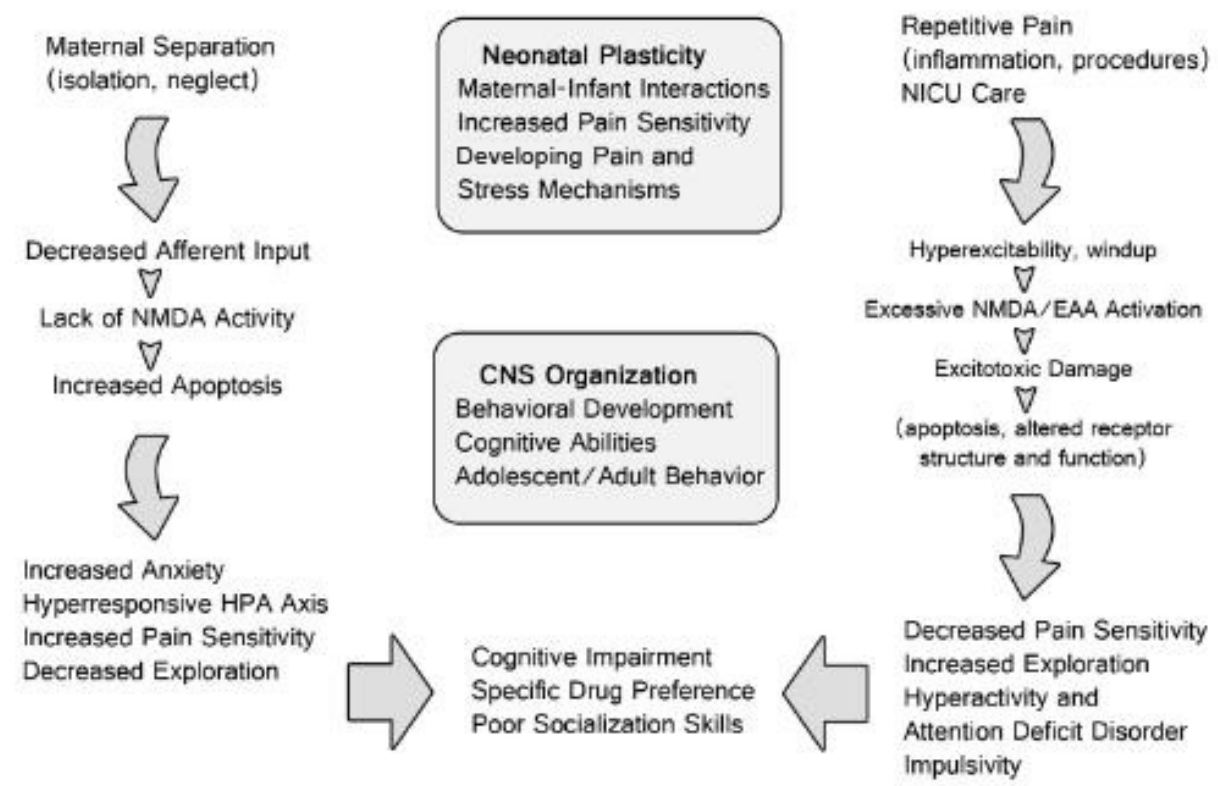

Fig. 1. Schematic diagram of the effects of neonatal pain and maternal separation in the neonate on brain plasticity and long term effects on subsequent brain development and behaviour

Vital parameters of clinical relevance for neonatal monitoring include body temperature, electrocardiogram (ECG), respiration, and blood oxygen saturation (Als, 1986; Polin \& Fox, 1992). Presently, body temperature is monitored with adhesive thermistors; ECG and respiration are obtained by adhesive skin electrodes. The oxygen saturation of the blood is monitored by a pulse oximeter with the sensor applied on the foot or palm of the neonate (Murković et al. 2003). Placement of these adhesive sensors and the presence of all the wires lead to discomfort and even painful stimuli when the electrodes have to be removed. Preterm infants, in particular the ones with an immature central nervous system, are highly sensitive for external stimuli such as noise, bright light, and pain. As the survival rate of neonates has increased significantly in the last decades (de Kleine et al., 2007), the quality of life of NICU graduates becomes an important issue as well. Alternative, non-invasive monitoring of vital physiological functions is a pressing need to provide convenient care and hence, may lead to improved developmental outcome of the neonates.

Recent advances in sensor technologies (Yang, 2006; Van Langenhove, 2007; Murković et al., 2003) and wireless communication technologies (Goldsmith, 2005) enable the creation of a 
new generation of healthcare monitoring systems with wearable electronics and photonics (Tao, 2005; Aarts \& Encarnação, 2006).

The Eindhoven University of Technology (TU/e) in the Netherlands has started a 10-year project on non-invasive perinatal monitoring in cooperation with the Máxima Medical Centre (MMC) in Veldhoven, the Netherlands. The goal of this collaboration is to improve the healthcare of the pregnant woman, and her child before, during, and after delivery. In the work on neonatal monitoring, we aim to integrate a multidisciplinary network of sensor technology, medical clinics and signal processing into revolutionary neonatal monitoring solutions (Chen et al., 2010b). The design skills needed range from medical science, human factors, material knowledge, smart textiles and form-giving to circuit design, user research, power management, signal processing and software engineering. Some intelligent designs have been developed covering different aspects of on non-invasive neonatal monitoring with wearable sensors, such as vital signs monitoring (Bouwstra et al, 2009; Chen, et al., 2010a; Chen, et al., 2010c), data transmission (Chen et al, 2009a), and power supply (Chen et al, 2008; Chen et al, 2009b). In this chapter, we present the design work of a smart jacket integrated with textile sensors and a power supply based on contactless energy transfer for neonatal monitoring.

The chapter is structured as follows. Section 2 explains the design process and design requirements. Section 3 describes the smart jacket design. Section 4 presents the wireless power supply design. Both section 3 and section 4 consist of the design concept, prototype implementation, and clinical testing or experimental results. Section 5 concludes the chapter.

\section{Design process and design requirements}

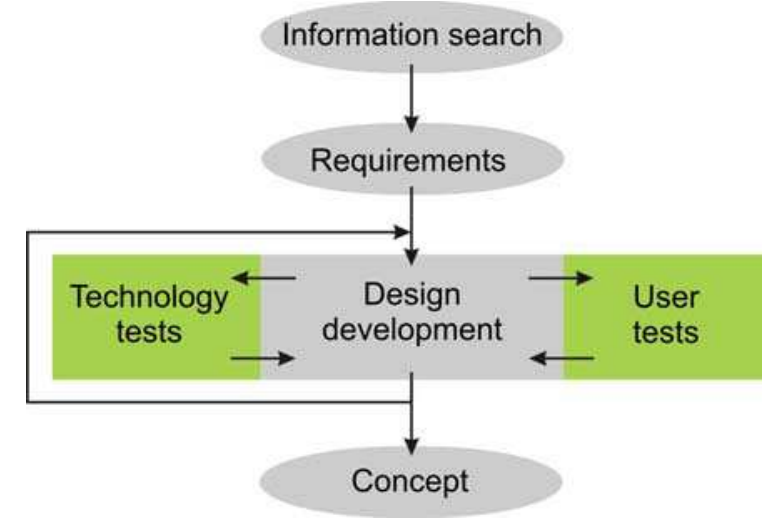

Fig. 2. Design process model

Methodologies from the field of Industrial Design are applied in the design process, which involves a unique integration of knowledge from medical science, design, and sensor technology. Fig. 2 shows the design process. The iterative process begins with an information search that includes user research involving doctors and nurses at MMC in Veldhoven and gathering of information on neonatal monitoring, smart textiles, power supply, etc.. Requirements were derived from the information search, forming a base for brainstorm sessions which resulted in ideas about technological challenges, functionality 
issues within NICU as well as form and senses. The ideas are then placed in a morphological diagram and combined to several initial concepts. Design choices are made through an iterative process in which proof of technology and user feedbacks provide clues for further development. The three aspects 'Technology, User Focus and Design', are strongly interwoven along the process.

With consideration of both user aspects and technical functions, the design should meet the following requirements:

- $\quad$ support the vital health monitoring functions

- be safe to use in the NICU environment

- be scalable to include more monitoring functions and local signal processing

- support continuous monitoring when the baby is inside the incubator or during Kangaroo mother care

- gain the feeling of trust by the parents and the medical staff through an attractive design

- be non-intrusive and avoid disturbance of the baby and avoid causes of stress

- $\quad$ provide appropriate feedback which is also interpretable for parents and hospital staff on whether the system's components are correctly functioning

- non-washable parts must be easy to remove

- look friendly, playful and familiar

\section{Smart jacket design for neonatal monitoring}

\subsection{Design concept}

The vision of the Neonatal Smart Jacket is a wearable unobtrusive continuous monitoring system realized by sensor networks and wireless communication, suitable for monitoring neonates inside the incubator and outside the incubator during Kangaroo mother care. The Neonatal Smart Jacket aims for providing reliable health monitoring as well as a comfortable clinical environment for neonatal care and parent-child interaction. The first step towards the Smart Jacket is the design of a jacket that:

1. contains the integration of conductive textiles for ECG monitoring,

2. forms a platform for future research, in which wireless communication, power supply and sensors are developed,

3. obtains a sense of trust by parents.

The concept of Diversity Textile Electrode Measurement (DTEM) is applied for the smart jacket design. The neonate wears a baby jacket that contains six conductive patches that sense biopotential signals at different positions to perform diversity measurements. Depending on the way the baby lies or is held, there are always patches that are in close contact with the skin because of pressure. When one sensor becomes loose from the skin, another sensor can provide a better signal. The system continuously measures which leads of the suit have superior contact and chooses the strongest signal for further processing. The concept offers a solution for skin contact, without jeopardizing comfort by tightness. It might also solve the problem of searching optimal electrode positions in the jacket, which varies per baby.

\subsection{Prototype}

A prototype jacket as shown in Fig. 3 was built according to the design requirements. The jacket is open at the front and has an open structure fabric on the back and hat, with the 
purpose of skin-on-skin contact, phototherapy and medical observation. The hat contains eye-protection and leaves room for future sensors. The aesthetics are designed to appear as regular baby clothing. The color combination of white and green with colorful happy animal heads is chosen because it is unisex while looking cheerful and clean.

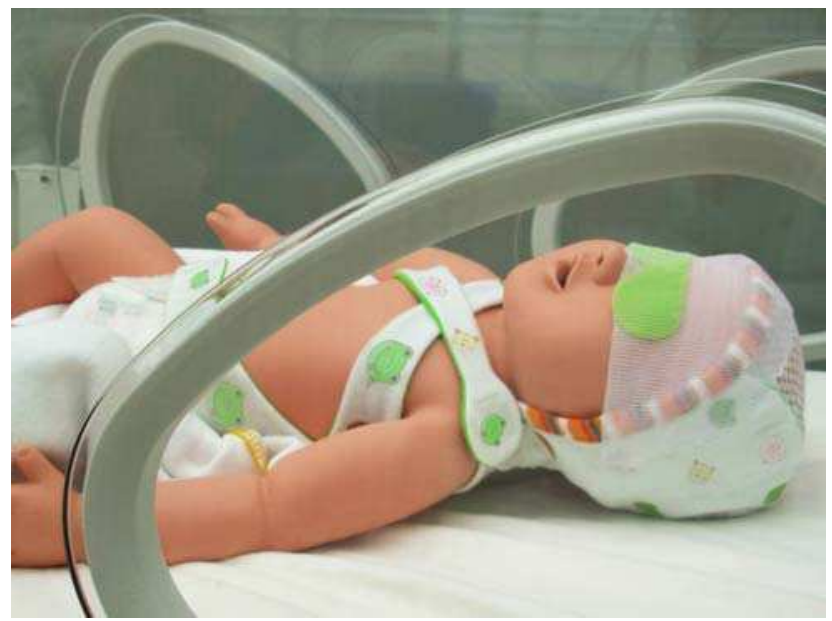

Fig. 3. Prototype smart jacket

The prototype is designed to have a stress-less dressing process as shown in Fig. 4: (1) the baby is laid down on the open jacket, (2) the lower belt is closed, (3) the hat is put on, and (4) finally the chest straps are closed.

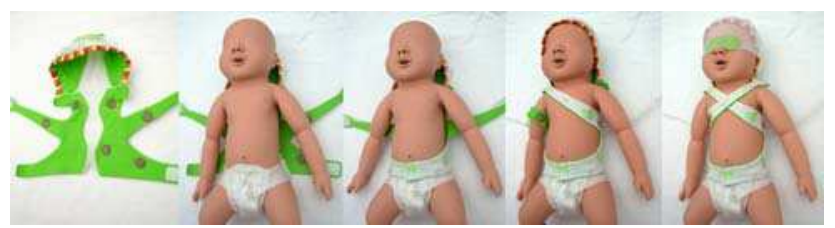

Fig. 4. Stress-less dressing process

Fig. 5 demonstrates the test patches with different versions of silver and gold textile electrodes and a blanket with large silver electrodes. The silver textile electrodes consist of silver plated nylons produced by Shieldex®. Construction details can be seen in Fig. 6 . Three layers (1) of cotton are used and on the middle layer (2) the circuit is sewn with Shieldex ${ }^{\circledR}$ silver plated yarn. On the first layer the electrode is sewn, stitching through the circuit on the middle layer (3). The electrode's connection to the monitor is realized by carbon wires obtained from regular disposable gel electrodes: the end of the carbon wires are stripped and sewn onto the circuit on the middle layer (4). (Carbon wire is a good alternative to metal buttons which are often applied, because it avoids the less stable soft-hard connection). Finally the third cotton layer for isolation is sewn to the others (5).

The gold printed electrodes consist of a thin smooth fiber with a metal print developed by TNO at Eindhoven, the Netherlands. The gold test patches are created in a similar way to the silver test patches, however in future application the circuit and electrode can be printed in one piece. 

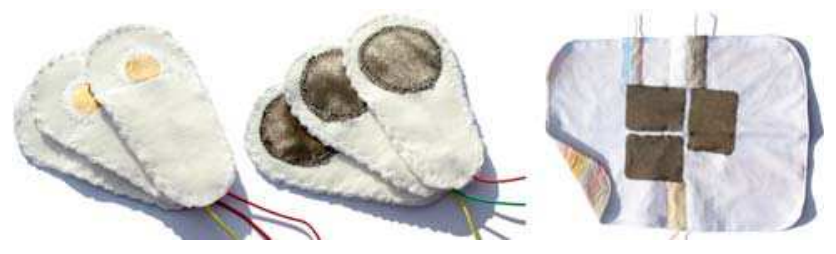

Fig. 5. Test patches and blanket
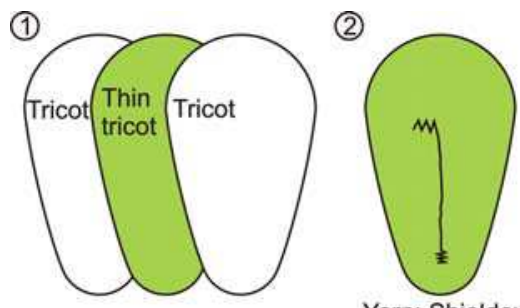

Yarn: Shieldex® 235/34 2-ply HC
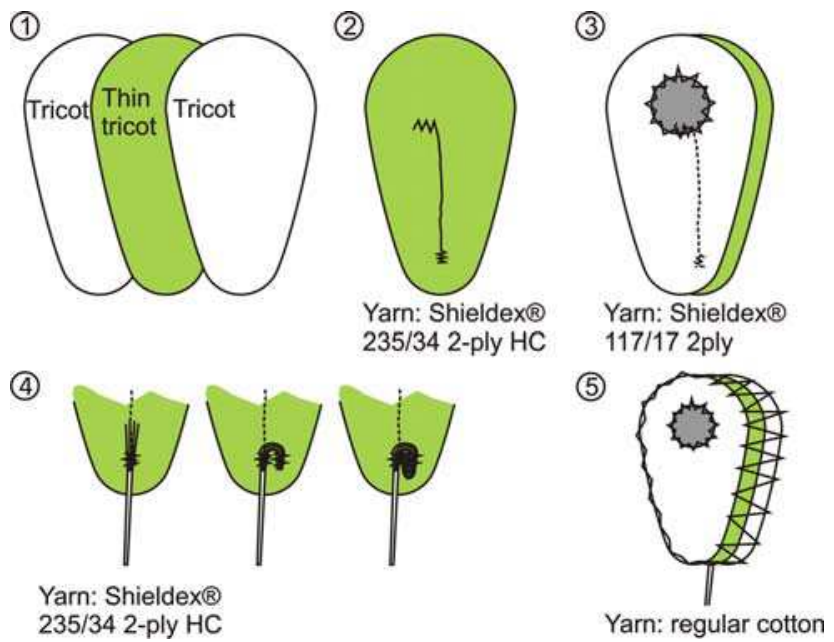

Fig. 6. Construction of textile electrodes

\subsection{Clinical testing}

Several experiments were carried out, ranging from experiments on adults as alternative subjects to neonates in the NICU at the MMC Veldhoven, the Netherlands. The goals are comparisons between the various textile electrodes, verification of their functioning on a neonate and verification of the DTEM concept. Finally, a wearability test of the jacket was performed.

An analysis of risks was performed before applying the prototypes to the NICU. Together with clinical physicists, a hospital hygiene and infection expert, and a neonatologist, the safety of the monitoring system and hygiene and allergy risks were analyzed. Precautions such as disinfection and allergy tests were taken. The ethical commission of the MMC Veldhoven approved the experiments.

First, we tested the quality of the ECG signals obtained by textile electrodes varying in material and size and gel electrodes (3M $\left.\mathrm{M}^{\mathrm{TM}} 2282 \mathrm{E}\right)$ are qualitatively compared. Fig. 7 shows the test setup. The electrodes were tested with two subjects: one neonate of 30 weeks and 5 days and one of 31 weeks and 6 days, both admitted in the NICU Veldhoven. The ECG is sensed by three textile electrodes in regular configuration and the data is acquired with a GE Heathcare Solar ${ }^{\circledR} 8000 \mathrm{M}$. The unprocessed digital data of derivation II was obtained from a network and imported and filtered in MATLAB. A notch, high pass and low pass filter are applied to remove the $50 \mathrm{~Hz}$ and higher harmonics, DC (direct current) component and high frequency noise. 


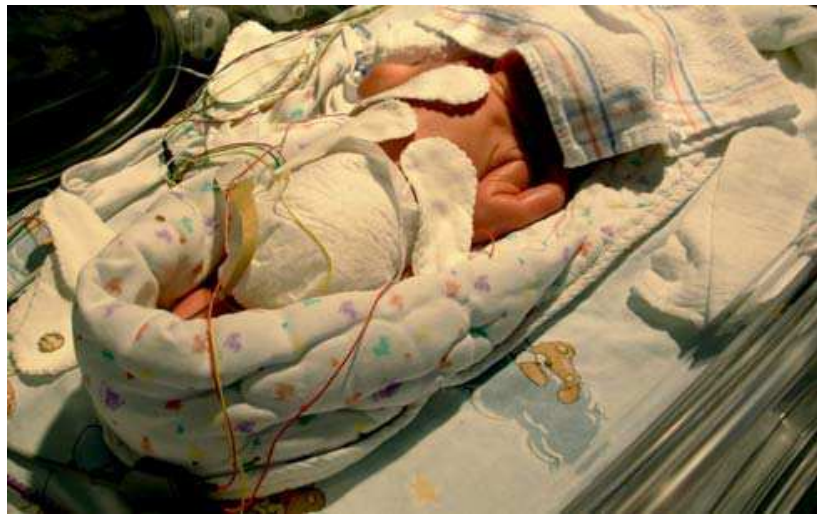

Fig. 7. Test setup

From Fig. 8 we can see that the quality of ECG obtained by the golden printed textile electrodes is good and the QRS complex can be seen clearly. The ECG curve in Fig. 8 is representative for the ECG quality by gold electrodes when the baby lies still.

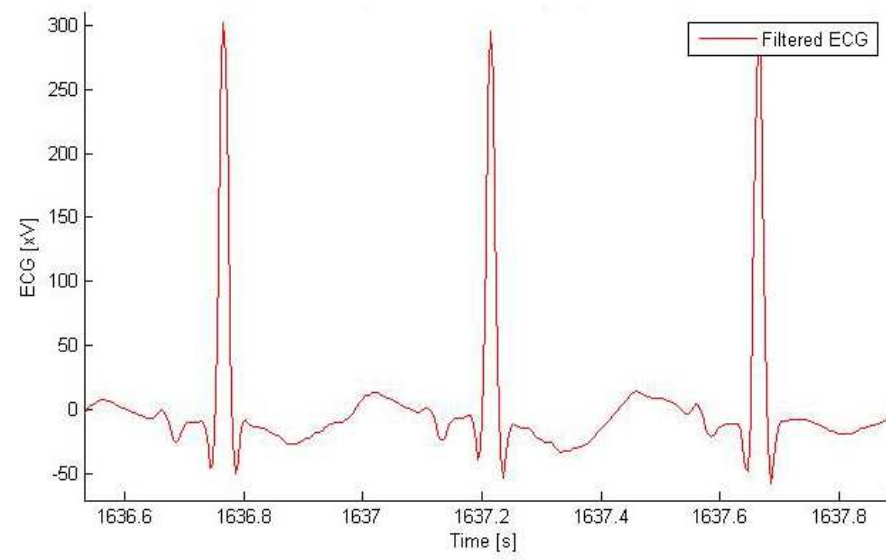

Fig. 8. Gold printed electrodes $\mathrm{D}=15 \mathrm{~mm}$

Secondly, we carried out tests to find out whether the concept of DTEM (Diversity Textile Electrode Measurement, see section 3.1) can improve the signal quality. The ECG obtained by large silver textile electrodes in a blanket where the neonate lies on, is compared to the ECG obtained by large silver patches held on the back. By this way, the effect of pressure by body weight can be investigated. From Fig. 9 we can see that the quality of ECG obtained by the silver textile electrodes is good and the QRS complex can be seen clearly as well. The shape of the ECG complex looks different from Fig. 8, because the heart is monitored from another angle.

Apart from reliable technology, the success of the Smart Jacket largely depends on the wearable comfort of the jacket. Tightness is desirable for sensor contact, although it might be in conflict with wearable comfort. Therefore, extra caution is taken by performing a 


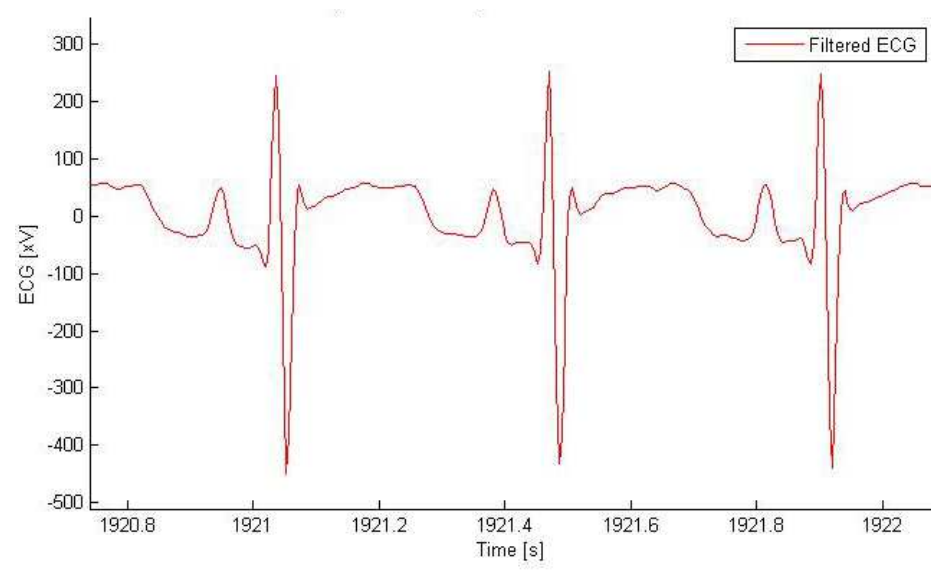

Fig. 9. Silver Shieldex®, 50mmx60mm, blanket

wearability test in an early design stage. Fig. 10 shows a stable neonate of 34 weeks being dressed in the first prototype of the Smart Jacket while being filmed. Compared to the stress that was caused when undressing the regular premature baby clothing, the dressing process of the Smart Jacket was very calm. The dressing time is about one minute. The model needs to be more adjustable in size due to large variations in proportions and range of dimensions: in the NICU neonates can grow from $500 \mathrm{~g}$ to $2000 \mathrm{~g}$ and body proportions vary especially when caused by medical conditions. The straps need to be improved for comfort in the next design iteration.

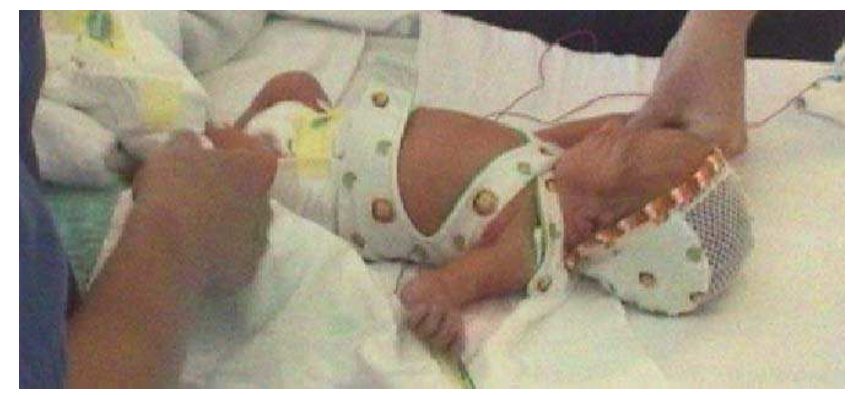

Fig. 10. Wearability test with the first prototype

\subsection{Discussion and improvements}

Due to the nature of conductive textiles, the quality of the ECG signal obtained with textile electrodes cannot exceed the gel electrodes: they are 'dry' electrodes with relatively loose skin contact and have a flexible structure that causes artifacts. However, the specific application of ECG monitoring neonates offers new design opportunities:

- A premature has smoother skin, which results in better skin contact

- The premature moves relatively little, which results in less movement artifacts

- The premature always lies or is being held, which offers continuous pressure, which leads to better skin contact 
Two textile electrode designs turn out very promising: (1) large $( \pm \mathrm{D}=40 \mathrm{~mm})$ silver plated textile electrodes and (2) small $( \pm D=15 \mathrm{~mm})$ gold printed electrodes. Both have different strengths and weaknesses. Large silver electrodes offer a stable ECG signal with low noise under the condition that pressure is applied. The silver seems hypoallergenic and does not change properties considerably after a few washing cycles.

The small gold printed electrodes, obtain a stable ECG signal with low noise, under the condition that pressure is applied in the beginning; once skin contact is established, little pressure is required. The gold print however is not hypoallergenic and looses conductivity after washing, due to corrosion of the metal layer beneath the gold. Although the silver electrodes could be applied without much adjustment, the gold prints are worth further development. They require less space due to higher conductivity, have a smoother surface that leads to better skin contact, are less flexible which leads to less artifacts and are seamless which leads to more comfort.

The monitoring of a neonate's ECG by diversity measurements realized by textile electrodes in the jacket definitely is a useful idea. Through experimental verification it is found that the quality of the ECG signal improves significantly due to a neonate's own body weight and is comparable to the quality of ECG signal obtained by gel electrodes.

Based on interviews with parents and medical staff, the conclusion can be drawn that the user groups are positive about the first results. They especially appreciate the freedom of movement, the aesthetic design, stress-less dressing process and integrated eye-protection. Improvements has been made on the design and a new version of the smart jacket has been developed as shown in Fig. 11.

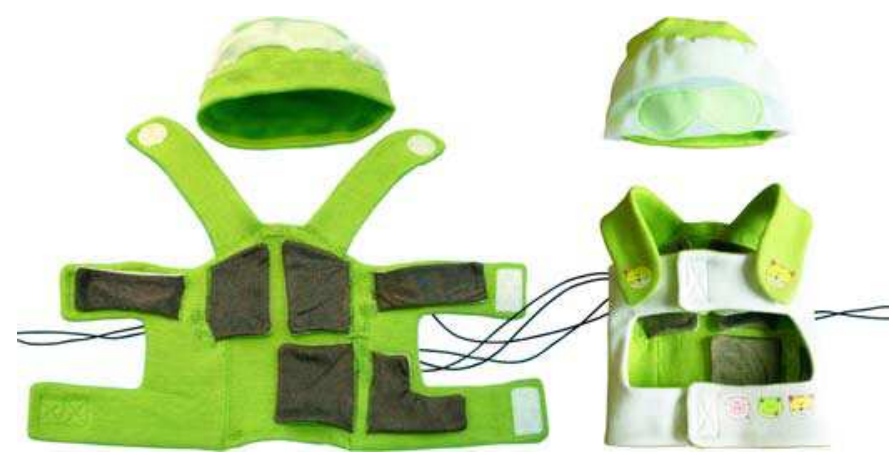

Fig. 11. New version of the smart jacket

The new version contains an extremely stretchable fabric that likely ensures adjustability to different sizes and proportions. The hat is kept separate for the same reasons. Furthermore, the straps are designed to prevent tightness around the neck. Large silver textile electrodes are applied in the new version. They are connected only on one of the four sides, in order to allow stretch of the jacket without stretch of the electrode itself. The medical staff and parents embrace the latest version of the smart jacket. At present this prototype is ready for further clinical testing within the MMC Veldhoven. The development of the Smart Jacket will be continued, initially by further development of the ECG sensors, wireless transmission and an adjustable size for different patients which enable clinical reliability tests. 


\section{Power supply design for neonatal monitoring}

\subsection{Design concept}

A key question for health monitoring with wearable sensors is how to obtain reliable electrical power for the sensors, signal amplifiers, filters and transmitters. The deployment of new sensing and monitoring devices for non-invasive healthcare and clinical applications requires design of the new power supplies. The power supply should be either long lasting or easy to recharge during usage (Tao, 2005) to perform near-real-time continuous monitoring. The need to minimize maintenance and replacement costs of batteries drives the development of innovative power solutions, encompassing energy scavenging (i.e. energy harvesting) technologies that exploit renewable and ambient sources of energy, such as solar energy, energy harvested from body heat and movement (Paradiso, 2005; Qin, 2008), and wireless power supplies (Catrysse, 2004; Ma, 2007).

Fundamental physiological parameters that should be continuously monitored during neonatal care are electrocardiogram (ECG), respiration, oxygen saturation of the blood $(\mathrm{O} 2-$ Sat), and body temperature. The amount of power required by different health monitoring sensors and processors is important for designing the power supply. We summarize the power consumption of monitoring and processing in Table 1. Based on the information of power consumption, our power supply should be able to deliver 150-200 $\mathrm{mW}$ for the health monitoring functions and more power is needed when charging batteries.

\begin{tabular}{|c|c|}
\hline Function & Power Consumption \\
\hline Data transmission & about $50 \mathrm{~mW}$ \\
\hline $\begin{array}{c}\text { ECG Read-out amplifier } \\
\text { for textile sensors }\end{array}$ & about $1 \mathrm{~mW}$ \\
\hline $\begin{array}{c}\text { body temperature } \\
\text { sensors }\end{array}$ & $50 \mathrm{~mW}$ \\
\hline SpO2 sensors & $45 \mathrm{~mW}$ \\
\hline Respiration sensors & below $1 \mathrm{~mW}$ \\
\hline
\end{tabular}

Table 1. Power consumption for monitoring and processing

With the above design requirements in mind, we come up with a technical solution and the concept of "PowerBoy", which uses contactless power and a rechargeable battery embedded in a plush toy for neonatal care. We propose to apply inductive energy transfer for the power supply due to its wireless feature and scalability. Inductive energy transfer will be employed for continuous power supply and for charging the battery when a neonate is lying inside the incubator. The rechargeable battery is used for energy storage and continuous power supply when the neonate is outside of incubator during Kangaroo mother care.

Fig. 12 shows an overview of the proposed system. In the system, a primary rectangular spiral winding, labelled $S_{A}$, is placed underneath a $60 \mathrm{~mm}$ thick incubator mattress. The primary winding forms part of a series resonant circuit driven by a half-bridge inverter and a power supply. The PowerBoy plush toy is equipped with, amongst other things, a secondary hexagon spiral winding, denoted $S_{B}$.

When the PowerBoy toy is placed on the mattress above the primary winding, the magnetic field is "picked-up" and an inductive link is formed. Power is then transferred from the primary winding to the secondary winding through their mutual inductance. A rectifier 


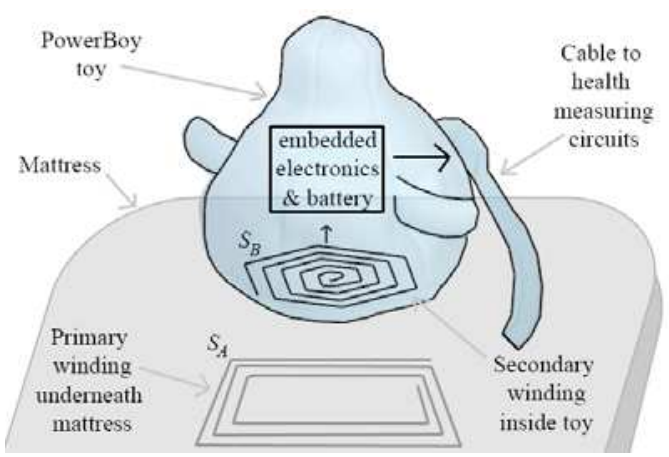

Fig. 12. An overview of the PowerBoy system

circuit and power converter charges a battery inside the toy, and supplies the monitoring equipment with power via a power cable, inside the toy's fluffy tail. When the baby and the PowerBoy toy are lifted up from the incubator, the inductive link is broken. The circuitry inside the toy detects this, and switches on the battery for powering the monitoring equipment. As the baby is laying down in the incubator again, and the PowerBoy toy placed in its correct position, inductive power is again restored and used for monitoring health parameters as well as charging the battery.

The power supply design focuses on the contactless energy transfer system as well as the primary and secondary windings that generate the magnetic fields. Afterwards, the mutual inductances are calculated and the power transfer equations solved to transfer the required amount of power. The magnetic field intensities are also estimated and discussed, as well as the battery charging circuitry.

\subsection{System design}

\subsubsection{Principle of contactless energy transfer}

Contactless Energy Transfer (CET) is the process in which elec $\neg$ trical energy is transferred between two or more electrical devices through inductive coupling as opposed to energy supply through conventional "plug and socket" connectors. The main method through which energy is transferred in the system is by magnetic fields and the mutual inductance between their primary and secondary coils (Sonntag, 2008). The CET system employs primary and secondary series resonance. This increases the efficiency. Fig. 13 shows a simplified schematic diagram of the CET circuit, which consists of two coils, forming a loosely coupled transformer. The primary coil generates a magnetic field, which is partly picked up by the secondary coil. The primary circuit and secondary circuit are separated by an air gap (incubator mattress).

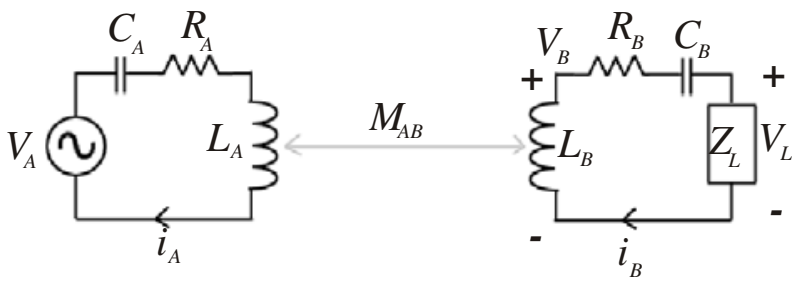

Fig. 13. Principle of inductive contactless energy transfer 
In this way, power can be transferred wirelessly. Assuming steady-state sinusoidal voltages and currents, the inductive link from Fig. 13 can be described mathematically by the following formulae:

$$
\begin{aligned}
& V_{A}=j \omega L_{A} i_{A}+i_{A} / j \omega C_{A}+R_{A} i_{A}-j \omega M_{A B} i_{B}, \\
& j \omega M_{A B} i_{A}=j \omega L_{B} i_{B}+i_{B} / j \omega C_{B}+R_{B} i_{B}+Z_{L} i_{B} .
\end{aligned}
$$

Here, $\omega$ is the radial frequency of the current. $V_{A}$ and $i_{A}$ are the primary voltage and current, respectively. The secondary current is given as $i_{B}$, and the induced secondary winding voltage is $V_{B} . R_{A}$ and $L_{A}$, and $R_{B}$ and $L_{B}$ are the internal resistances and self inductances of the primary and secondary windings, $S_{A}$ and $S_{B}$, respectively. The mutual inductance between the primary and secondary winding is denoted as $M_{A B} . C_{A}$ and $C_{B}$ are the primary and secondary resonance capacitors, respectively. $Z_{L}$ represents the secondary equivalent load impedance and $V_{L}$ the voltage over the load.

\subsubsection{Primary and secondary windings}

The primary and secondary CET windings play a vital role in determining the power transfer capability of the system. The size of the secondary winding is chosen so that it can fit into the bottom of the PowerBoy toy. A two layer hexagon spiral winding with a radius of $40 \mathrm{~mm}$ is used. The primary coil is a rectangular spiral winding with $120 \mathrm{~mm}$ length and $100 \mathrm{~mm}$ width. The primary and secondary windings are shown in Fig. 14 (a) and (b). Table 2 summarizes their physical dimensions and electrical properties.

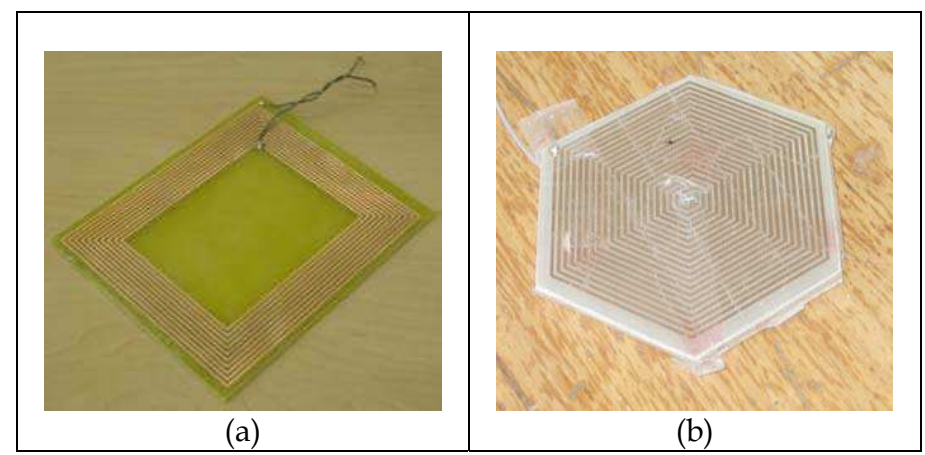

Fig. 14. (a) Primary rectangular spiral winding, and (b) secondary hexagon spiral winding

\subsubsection{Mutual inductance values \& calculated power transfer}

The mutual inductance between the primary and secondary windings, as shown in equations (1) and (2), is vital in calculating the secondary windings' induced voltage and the power transfer capability of the system. Using finite element analysis software (Maxwell 3D version 11, Ansoft Corporation) the primary and secondary windings are simulated using a three-dimensional environment. The mutual inductance between the windings is estimated using the magneto-static solution type. Fig. 15 shows a three-dimensional image of the mutual inductance results. The results show a maximum mutual inductance of $1.32 \mu \mathrm{H}$ when the secondary winding is centred directly above the primary winding, i.e. the best- 


\begin{tabular}{|c|c|c|}
\hline Parameter & $\begin{array}{c}\text { Primary Winding } \\
\text { Value }\end{array}$ & $\begin{array}{c}\text { Secondary Winding } \\
\text { Value }\end{array}$ \\
\hline Dimensions & $100 \mathrm{~mm} \times 120 \mathrm{~mm}$ & $40 \mathrm{~mm}$ radius \\
\hline Turns per layer & 10 turns & 19 turns \\
\hline Layers & 1 & 2 \\
\hline Thickness & $100 \mu \mathrm{m}$ & $100 \mu \mathrm{m}$ \\
\hline Track width & $1 \mathrm{~mm}$ & $1 \mathrm{~mm}$ \\
\hline Track spacing & $1 \mathrm{~mm}$ & $0.5 \mathrm{~mm}$ \\
\hline Inductance & $17.5 \mu \mathrm{H}$ & $34.56 \mu \mathrm{H}$ \\
\hline Resistance (DC) & $2.48 \Omega$ & $3.34 \Omega$ \\
\hline Resistance (2.5 MHz) & $3.47 \Omega$ & $8.80 \Omega$ \\
\hline
\end{tabular}

Table 2. Physical dimensions \& electrical properties of the primary and secondary windings

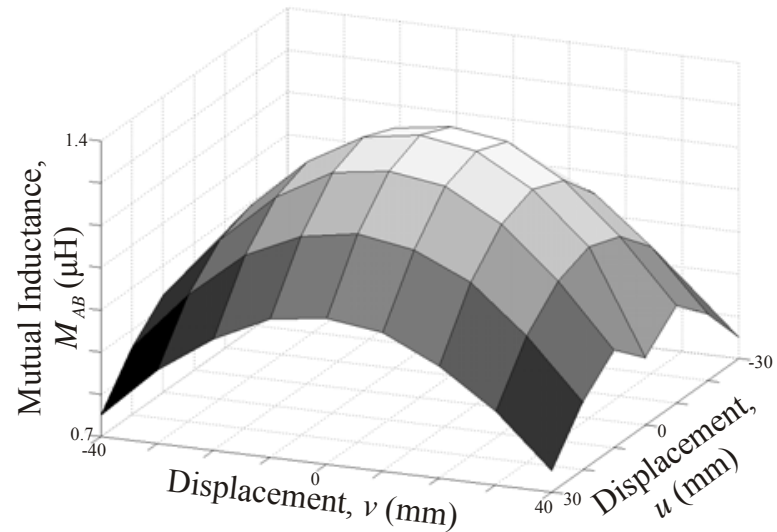

Fig. 15. A three-dimensional image of the mutual inductance results

case secondary winding and the preferred PowerBoy toy placement. The worst-case mutual inductance occurs when the secondary winding is placed close to the corners of the primary winding. At these positions, the mutual inductance is approximately $0.75 \mu \mathrm{H}$. This is the furthest distance the PowerBoy toy may be placed from the primary winding, to still operate normally.

The CET system should be able to power a $840 \mathrm{~mW}$ equivalent load impedance. This takes into account the $200 \mathrm{~mW}$ for the health monitoring systems, and $500 \mathrm{~mW}(100 \mathrm{~mA} @ 5 \mathrm{~V})$ for charging the battery. An extra $20 \%$ is added to compensate for any unforeseen losses. The power transfer equations are solved in equation (1) and (2) by making sure that the system can power the maximum load at the worst-case winding placement, so that it will guarantee normal operation and transfer of power for the system, at any toy position within the primary winding area. Table 3 shows the calculated primary currents, secondary currents and load voltages, for the worst-case and best-case toy placements, for three different power transfer scenarios. Firstly, for a fully charged battery, only $200 \mathrm{~mW}$ load power is required for the health monitoring systems. Secondly, for a partially charged battery, $450 \mathrm{~mW}$ is required (i.e. $200 \mathrm{~mW}$ health monitoring system $+250 \mathrm{~mW}$ for half the battery charging power). Thirdly, for a completely discharged battery, the full $700 \mathrm{~mW}$ is transferred. From 
Table 3, it can be seen that for a certain load power, the best-case PowerBoy toy placement has a higher induced voltage than the worst-case placement.

\begin{tabular}{|c|c|c|}
\hline $\begin{array}{c}\text { Load power } \\
\text { value }\end{array}$ & $\begin{array}{c}\text { Best PowerBoy toy } \\
\text { placement }\end{array}$ & $\begin{array}{c}\text { Worst PowerBoy } \\
\text { toy placement }\end{array}$ \\
\hline & $i_{A}=1.53 \mathrm{~A}$ (peak) & $i_{A}=1.53 \mathrm{~A}$ (peak) \\
$200 \mathrm{~mW}$ & $i_{B}=13 \mathrm{~mA}$ (peak) & $i_{B}=23 \mathrm{~mA}$ (peak) \\
& $V_{L}=31.1 \mathrm{~V}$ (peak) & $V_{L}=17.5 \mathrm{~V}$ (peak) \\
\hline & $i_{A}=1.42 \mathrm{~A}$ (peak) & $i_{A}=1.42 \mathrm{~A}$ (peak) \\
$450 \mathrm{~mW}$ & $i_{B}=31 \mathrm{~mA}$ (peak) & $i_{B}=57 \mathrm{~mA}$ (peak) \\
& $V_{L}=29 \mathrm{~V}$ (peak) & $V_{L}=16 \mathrm{~V}$ (peak) \\
\hline & $i_{A}=1.29 \mathrm{~A}$ (peak) & $i_{A}=1.27 \mathrm{~A}$ (peak) \\
$700 \mathrm{~mW}$ & $i_{B}=54 \mathrm{~mA}$ (peak) & $i_{B}=100 \mathrm{~mA}$ (peak) \\
& $V_{L}=25.9 \mathrm{~V}$ (peak) & $V_{L}=13.8 \mathrm{~V}$ (peak) \\
\hline
\end{tabular}

Table 3. Power transfer results for different winding placements and load power

\subsubsection{Magnetic field values}

The magnetic fields created by the currents circulating in the primary and secondary windings are estimated using finite element analysis software (Maxwell 3D version 11, Ansoft Corporation) and solving the fields using the magneto-static solution type. According to (ICNRP, 1998), the exposure to time-varying magnetic field values at a frequency of $2.4576 \mathrm{MHz}$ (the optimum operating frequency for the proposed system) is safe for general public exposure, at approximately $0.3 \mathrm{~A} / \mathrm{m}$ (RMS) and less. The results from the magnetic field estimation show that the magnetic field produced by the primary winding has a maximum value of $4.2 \mathrm{~A} / \mathrm{m}$ on the surface of the mattress. The magnetic field intensity reaches a value of $0.3 \mathrm{~A} / \mathrm{m}$ at a radius of approximately $155 \mathrm{~mm}$ from the centre of the winding. The magnetic field from the secondary winding is mostly contained inside the PowerBoy toy and is negligible outside the toy. Thus, for safety reasons, it is advisable to place the baby at least $155 \mathrm{~mm}$ away from the centre of the primary winding.

\subsubsection{Battery charging circuit}

The battery charging circuit comprises of a rechargeable $2400 \mathrm{mAh} 3.6 \mathrm{~V}$ NiMH battery and a battery charging circuit. The battery charging current is limited $100 \mathrm{~mA}$. A fully discharged battery will thus take approximately 24 hours to charge. The battery has the ability to power the $200 \mathrm{~mW}$ health monitoring circuits for approximately 40 hours.

\subsection{Prototype}

A prototype was built to demonstrate the performance of the proposed power supply. The users of the power supply will be hospital staff (e.g. doctors, nurses and technicians) working at NICUs in hospitals, as well as parents and the neonates under monitoring. Therefore, we take the aspects of aesthetics and user friendliness into our design. The PowerBoy power supply system consists of a PowerBoy toy, a PowerBoy house and a soft sheet as shown in Fig. 16. In this subsection, the details of the electronics in the prototype are presented. 
The prototype is implemented modularly, and contains eight major sub-systems as shown in the block diagram in Fig. 17. Here the black arrows indicate the flow of power, while the grey arrows show magnetic fields.

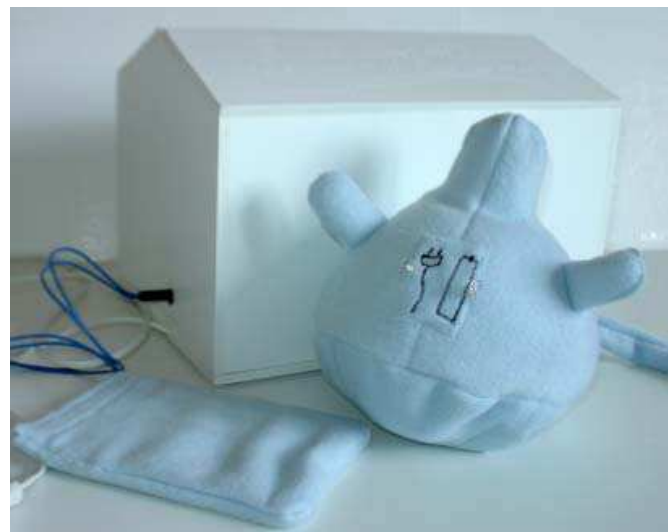

Fig. 16. The PowerBoy system, consisting of a toy, a house and a soft sheet

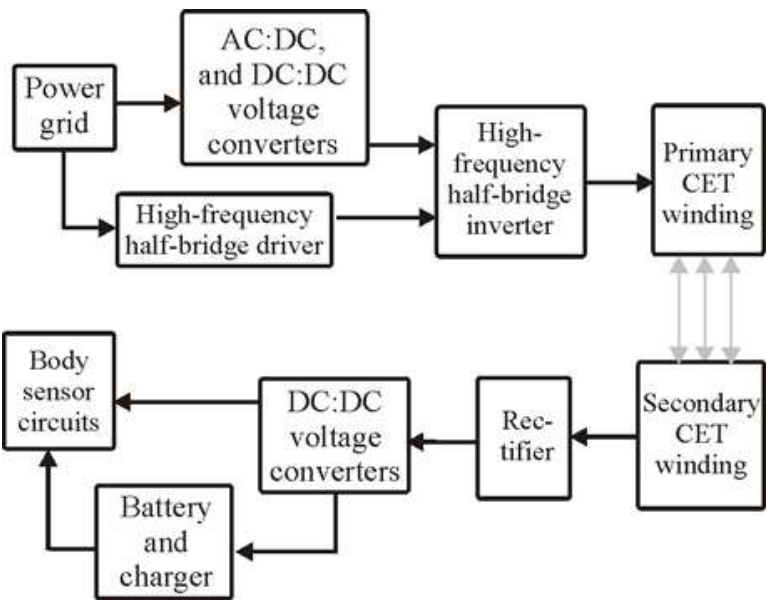

Fig. 17. Block diagram of CET power supply

Firstly, integrated into the PowerBoy house, is the circuitry used to generate the required voltages and signals used in the contactless energy transfer system. This includes three ACto-DC power converters, for converting the $230 \mathrm{~V}, 50 \mathrm{~Hz}$ mains voltage into $+9 \mathrm{~V},-9 \mathrm{~V}$ and $24 \mathrm{~V}$ (DC), respectively. Additionally, it contains a DC-to-DC converter which generated a $3.3 \mathrm{~V}$ (DC), a $2.4576 \mathrm{MHz}$ oscillator (XO53B-2.4576M) a half-bridge inverter (using two IRF510 N-channel MOSFETS) and a high-frequency MOSFET driver, based on the designs in (Sonntag, 2008). This house encloses the PCBs of the drive circuit and the power supply box. Fig. 18 gives a top view of the drive circuits in the PowerBoy housing. In this manner the system can become portable. 


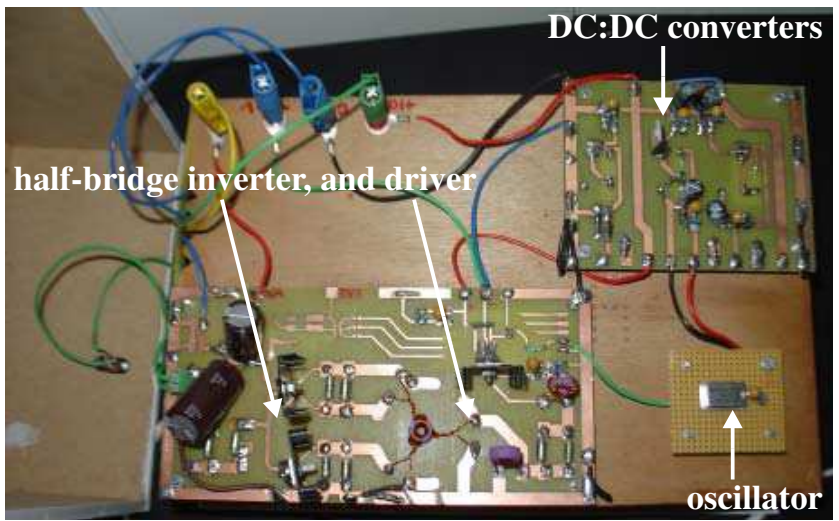

Fig. 18. Top view of the drive circuit in the PowerBoy house

Secondly, is the PowerBoy toy as shown in Fig. 19 (a): Integrated into the toy is the secondary winding (on the bottom). Additionally, it contains the rectifier circuit, a voltage converter and the battery charging circuits. The PowerBoy is designed to be a friendly companion for the neonates and is made from soft materials which are stitched together, to make a spherical-shaped toy. A process of participatory de-sign was followed for the formgiving and material choosing. On the chest of the toy are two LEDs which indi-cate the status of the power supply and the battery. When CET power is available, the left LED next to the power-plug icon lights up. When the PowerBoy is picked up and the battery is used, the right side LED next to the battery icon lights up. The battery charg-ing circuitry as shown in Fig. 19 (b) is based on the design given in (Hayles, 2008) and consists of a programmed PIC17C711 microprocessor and a controlled current source using a LM317 voltage regulator and a $\mathrm{BC} 548$ transistor.

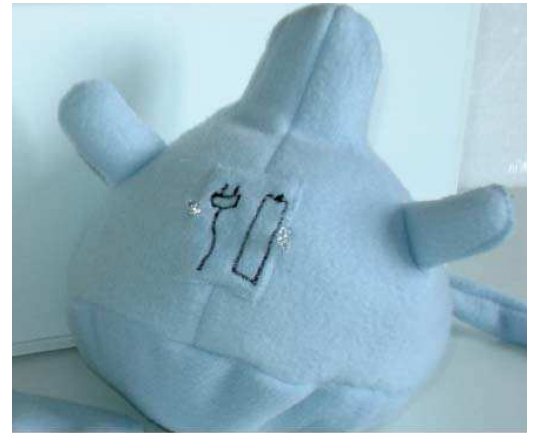

(a)

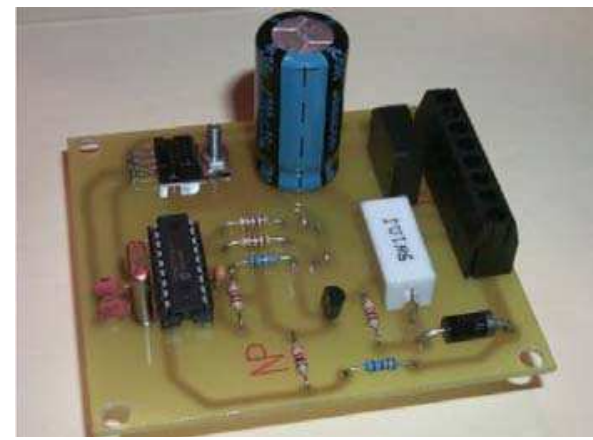

(b)

Fig. 19. (a) PowerBoy toy and (b) battery charging circuit

Thirdly, the primary winding is integrated into a soft material pocket called the soft sheet. This sheet softens the hard edges of the PCB containing the primary winding. It does not come in to contact with the baby but it feels and looks friendlier when inter-acting with it. This sheet is positioned underneath the mattress. 
Instead of an additional technical device in the incubator, PowerBoy is an attractive alternative with its baby-friendly appearance. Parents will appreciate this design, and may experience some relief of tension.

\subsection{Experimental results}

To verify the power transfer calculations and results, several power transfer experiments are preformed. Fig. 20 draws the implemented circuits for the prototype and experiments. Here, $T_{1}$ and $T_{2}$ are the two MOSFETS used in the half-bridge inverter, and $V_{A A}$ is its input voltage. The final output voltage- and current to the neonatal health monitoring system is $V_{O}$ and $I_{O}$, respectively.

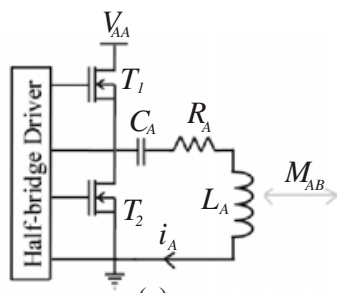

(a)

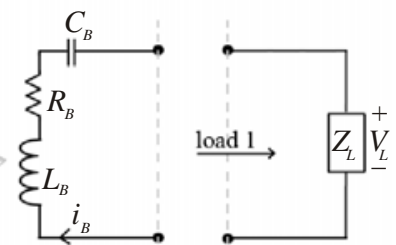

(b)

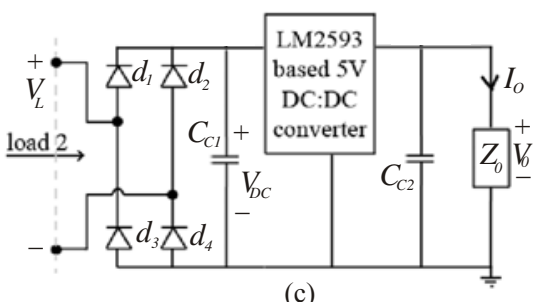

(c)

Fig. 20. The implemented (a) primary circuit, (b) the secondary test circuit with only a resistor as load, and (c) the rectifier, DC:DC converter and resistor as load.

The measurements are preformed by placing the centre of the secondary winding at discrete positions above the primary winding, at a height of $z=65 \mathrm{~mm}$. Due to the symmetry in the primary winding, only nine positions, as shown in Fig. 21, are measured.

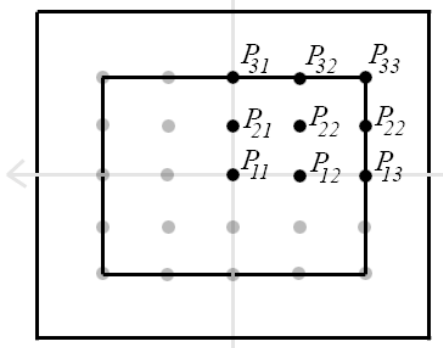

Fig. 21. The measurement positions above the primary winding 
Firstly, the system is implemented with the primary circuit (a) and secondary circuit (b) as shown in Fig. 20. The peak secondary load voltage, $V_{L}$, is measured for a no-load situation $\left(Z_{L} \rightarrow \infty\right)$. The primary current of $1.28 \mathrm{~A}$ (peak) is achieved by driving the half-bridge inverted with a voltage of, $V_{A A}=23.5 \mathrm{~V}$. Fig. 22 illustrates a graph with a clear peak at the centre. This confirms the mutual inductance maximum at this point. The maximum secondary induced voltage is $26.5 \mathrm{~V}$ (peak) and the minimum is $13.78 \mathrm{~V}$ (peak).

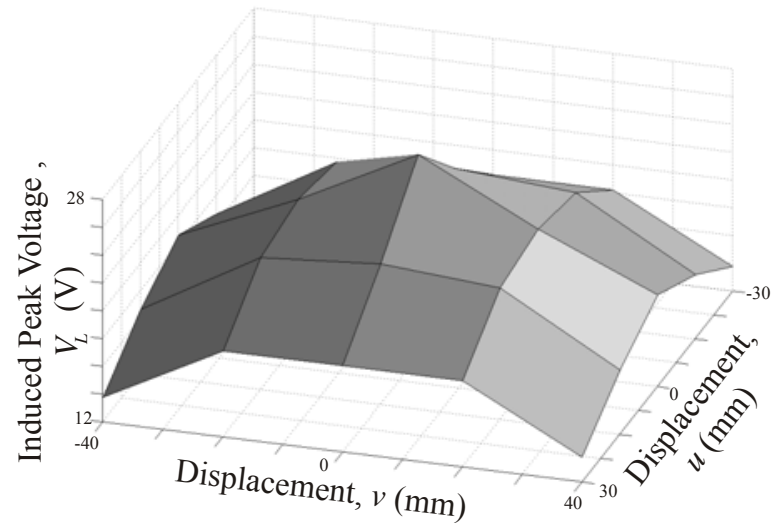

Fig. 22. The peak induced voltage

Secondly, the primary current, secondary current, and load voltage is measured using a load resistance of $Z_{L}=85.8 \Omega$. This corresponds to an $840 \mathrm{~mW}$ power transfer at the worst-case secondary winding placement $\left(P_{33}\right.$ on Fig. 21). With $V_{A A}=23.5 \mathrm{~V}$, the results are shown in Table 4. From Table 4, we can see that at the worst-case secondary winding placement, the system is capable of transferring the needed $840 \mathrm{~mW}$ at approximately $12 \mathrm{~V}$ (peak).

\begin{tabular}{|c|c|c|c|c|}
\hline $\begin{array}{c}\text { Secondary } \\
\text { winding } \\
\text { position }\end{array}$ & $\begin{array}{c}\text { Primary } \\
\text { winding } \\
\text { current } \\
\boldsymbol{i}_{A} \text { (peak) }\end{array}$ & $\begin{array}{c}\text { Secondary } \\
\text { winding } \\
\text { current } \\
\boldsymbol{i}_{\boldsymbol{B}} \text { (peak) }\end{array}$ & $\begin{array}{c}\text { Load } \\
\text { voltage } \\
\boldsymbol{V}_{\boldsymbol{L}} \\
\text { (peak) }\end{array}$ & $\begin{array}{c}\text { Load } \\
\text { power } \\
\boldsymbol{P}_{\boldsymbol{L}}\end{array}$ \\
\hline $\boldsymbol{P}_{11}$ & $1.08 \mathrm{~A}$ & $185 \mathrm{~mA}$ & $16.55 \mathrm{~V}$ & $1.53 \mathrm{~W}$ \\
\hline $\boldsymbol{P}_{12}$ & $1.08 \mathrm{~A}$ & $184 \mathrm{~mA}$ & $16.0 \mathrm{~V}$ & $1.47 \mathrm{~W}$ \\
\hline $\boldsymbol{P}_{13}$ & $1.25 \mathrm{~A}$ & $156 \mathrm{~mA}$ & $13.7 \mathrm{~V}$ & $1.07 \mathrm{~W}$ \\
\hline $\boldsymbol{P}_{21}$ & $1.10 \mathrm{~A}$ & $185 \mathrm{~mA}$ & $16.0 \mathrm{~V}$ & $1.48 \mathrm{~W}$ \\
\hline $\boldsymbol{P}_{22}$ & $1.15 \mathrm{~A}$ & $177 \mathrm{~mA}$ & $15.5 \mathrm{~V}$ & $1.37 \mathrm{~W}$ \\
\hline $\boldsymbol{P}_{23}$ & $1.25 \mathrm{~A}$ & $176 \mathrm{~mA}$ & $13.5 \mathrm{~V}$ & $1.19 \mathrm{~W}$ \\
\hline $\boldsymbol{P}_{31}$ & $1.22 \mathrm{~A}$ & $180 \mathrm{~mA}$ & $14.0 \mathrm{~V}$ & $1.26 \mathrm{~W}$ \\
\hline $\boldsymbol{P}_{32}$ & $1.22 \mathrm{~A}$ & $180 \mathrm{~mA}$ & $13.5 \mathrm{~V}$ & $1.22 \mathrm{~W}$ \\
\hline $\boldsymbol{P}_{33}$ & $1.16 \mathrm{~A}$ & $150 \mathrm{~mA}$ & $11.7 \mathrm{~V}$ & $878 \mathrm{~mW}$ \\
\hline
\end{tabular}

Table 4. Experimental results of $840 \mathrm{~mW}$ power transfer 
Thirdly, experiments are conducted with the implementation of the secondary circuit (c) as shown in Fig. 20. Simulating a fully charged battery (a battery charger is not drawing any current), a load power of $200 \mathrm{~mW}$ is required. With an expected load voltage, $V_{O}=5 \mathrm{~V}$ (DC), an equivalent load resistance of $125 \Omega(126 \Omega$ implemented) is used. The expected load current is $I_{O}=39.7 \mathrm{~mA}$. With $V_{A A}=23.5 \mathrm{~V}$, the primary and secondary winding currents, the rectifier voltage, $V_{D C}$, and the load voltage $V_{O}$, are measured. Table 5 shows that the load voltage of 5 $\mathrm{V}$, and consequently $200 \mathrm{~mW}$ load power, was maintained at all the measuring positions.

\begin{tabular}{|c|c|c|c|c|}
\hline $\begin{array}{c}\text { Secondary } \\
\text { winding } \\
\text { position }\end{array}$ & $\begin{array}{c}\text { Primary } \\
\text { winding } \\
\text { current } \\
\boldsymbol{i}_{A} \text { (peak) }\end{array}$ & $\begin{array}{c}\text { Secondary } \\
\text { winding } \\
\text { current } \\
\boldsymbol{i}_{B} \text { (peak) }\end{array}$ & $\begin{array}{c}\text { Rectifier } \\
\text { Voltage } \\
\boldsymbol{V}_{D C} \\
(\mathbf{D C})\end{array}$ & $\begin{array}{c}\text { Load } \\
\text { Voltage } \\
\boldsymbol{V}_{\boldsymbol{O}} \\
\text { (DC) }\end{array}$ \\
\hline $\boldsymbol{P}_{11}$ & $1.30 \mathrm{~A}$ & $48 \mathrm{~mA}$ & $17.6 \mathrm{~V}$ & $5 \mathrm{~V}$ \\
\hline $\boldsymbol{P}_{12}$ & $1.32 \mathrm{~A}$ & $48 \mathrm{~mA}$ & $16.7 \mathrm{~V}$ & $5 \mathrm{~V}$ \\
\hline $\boldsymbol{P}_{13}$ & $1.26 \mathrm{~A}$ & $55 \mathrm{~mA}$ & $12.5 \mathrm{~V}$ & $5 \mathrm{~V}$ \\
\hline $\boldsymbol{P}_{21}$ & $1.28 \mathrm{~A}$ & $50 \mathrm{~mA}$ & $16 \mathrm{~V}$ & $5 \mathrm{~V}$ \\
\hline $\boldsymbol{P}_{22}$ & $1.26 \mathrm{~A}$ & $50 \mathrm{~mA}$ & $15 \mathrm{~V}$ & $5 \mathrm{~V}$ \\
\hline $\boldsymbol{P}_{23}$ & $1.28 \mathrm{~A}$ & $58 \mathrm{~mA}$ & $11.7 \mathrm{~V}$ & $5 \mathrm{~V}$ \\
\hline $\boldsymbol{P}_{31}$ & $1.28 \mathrm{~A}$ & $52 \mathrm{~mA}$ & $13.3 \mathrm{~V}$ & $5 \mathrm{~V}$ \\
\hline $\boldsymbol{P}_{32}$ & $1.28 \mathrm{~A}$ & $50 \mathrm{~mA}$ & $12.5 \mathrm{~V}$ & $5 \mathrm{~V}$ \\
\hline $\boldsymbol{P}_{33}$ & $1.30 \mathrm{~A}$ & $59 \mathrm{~mA}$ & $9.6 \mathrm{~V}$ & $5 \mathrm{~V}$ \\
\hline
\end{tabular}

Table 5. Experimental results of power transfer under the condition of fully charged battery Fourthly, simulating a completely drained battery, a load power of $700 \mathrm{~mW}$ is required (200 $\mathrm{mW}$ for the health monitoring circuits and $500 \mathrm{~mW}$ for the battery charging). The equivalent load resistor of $35.7 \Omega\left(36.1 \Omega\right.$ implemented) is used. The expected load current is $I_{O}=139$ $\mathrm{mA}$ ). With $V_{A A}=23.5 \mathrm{~V}$, the primary and secondary winding currents, the rectifier voltage, $V_{D C}$, and the load voltage $V_{O}$, are measured. Table 6 shows the results.

\begin{tabular}{|c|c|c|c|c|}
\hline $\begin{array}{c}\text { Secondary } \\
\text { winding } \\
\text { position }\end{array}$ & $\begin{array}{c}\text { Primary } \\
\text { winding } \\
\text { current } \\
\boldsymbol{i}_{A} \text { (peak) }\end{array}$ & $\begin{array}{c}\text { Secondary } \\
\text { winding } \\
\text { current } \\
\boldsymbol{i}_{\boldsymbol{B}} \text { (peak) }\end{array}$ & $\begin{array}{c}\text { Rectifier } \\
\text { Voltage } \\
\boldsymbol{V}_{\boldsymbol{D C}} \\
\text { (DC) }\end{array}$ & $\begin{array}{c}\text { Load } \\
\text { Voltage } \\
\boldsymbol{V}_{\boldsymbol{O}} \\
\text { (DC) }\end{array}$ \\
\hline $\boldsymbol{P}_{11}$ & $1.10 \mathrm{~A}$ & $158 \mathrm{~mA}$ & $14 \mathrm{~V}$ & $5 \mathrm{~V}$ \\
\hline $\boldsymbol{P}_{12}$ & $1.13 \mathrm{~A}$ & $160 \mathrm{~mA}$ & $13.5 \mathrm{~V}$ & $5 \mathrm{~V}$ \\
\hline $\boldsymbol{P}_{13}$ & $1.17 \mathrm{~A}$ & $184 \mathrm{~mA}$ & $9.9 \mathrm{~V}$ & $5 \mathrm{~V}$ \\
\hline $\boldsymbol{P}_{21}$ & $1.14 \mathrm{~A}$ & $170 \mathrm{~mA}$ & $12.2 \mathrm{~V}$ & $5 \mathrm{~V}$ \\
\hline $\boldsymbol{P}_{22}$ & $1.14 \mathrm{~A}$ & $170 \mathrm{~mA}$ & $12.2 \mathrm{~V}$ & $5 \mathrm{~V}$ \\
\hline $\boldsymbol{P}_{23}$ & $1.18 \mathrm{~A}$ & $194 \mathrm{~mA}$ & $8.8 \mathrm{~V}$ & $5 \mathrm{~V}$ \\
\hline $\boldsymbol{P}_{31}$ & $1.17 \mathrm{~A}$ & $182 \mathrm{~mA}$ & $10.4 \mathrm{~V}$ & $5 \mathrm{~V}$ \\
\hline $\boldsymbol{P}_{32}$ & $1.18 \mathrm{~A}$ & $190 \mathrm{~mA}$ & $10 \mathrm{~V}$ & $5 \mathrm{~V}$ \\
\hline $\boldsymbol{P}_{33}$ & $1.18 \mathrm{~A}$ & $200 \mathrm{~mA}$ & $6.7 \mathrm{~V}$ & $5 \mathrm{~V}$ \\
\hline
\end{tabular}

Table 6. Experimental Results Of Power Transfer under the condition of completely drained battery 
These results show that the load voltage of $5 \mathrm{~V}$, and consequently $700 \mathrm{~mW}$ load power, was maintained at all the measuring positions. The system is thus capable of charging a completely discharged battery, while providing $200 \mathrm{~mW}$ of power to the neonatal health monitoring circuit, and still maintaining a $5 \mathrm{~V}$ (DC) output voltage.

\subsection{Discussion}

The proposed power supply satisfies the requirements of neonatal monitoring and provides continuous power when the neonate is inside the incubator or during Kangaroo mother care. The PowerBoy prototype was designed and implemented to demonstrate the performance of the power supply and the possibilities for aesthetic features. Experimental results showed that the prototype transfers approximately $840 \mathrm{~mW}$ of power. To evaluate the PowerBoy concept with user feedback, we had meetings with the group leader of the NICU at MMC, Prof. dr. Sidarto Bambang Oetomo and the head of the NICU nurses, Astrid Osagiator. They were enthusiastic about the concept and prototype. Further improvements and clinical verification will be conducted at MMC to integrate the power supply into the non-invasive neonatal monitoring systems.

New development of CET has the potential to enable automatic location detection and power switching, consequently, automatic power management with less magnetic fields can be foreseen for neonatal monitoring when the baby is at different locations inside the incubator.

Due to the amount of energy consumption of current sensor technologies, it is not yet feasible to harvest enough power from the NICU environment. Further development on sensors and components with low power consumption could bring opportunities for energy harvesting technologies to support neonatal monitoring.

\section{Conclusion}

In this chapter we presented the design of a smart jacket and the design of a power supply for neonatal monitoring with wearable sensors. These are examples of what can be done now, in the first decade of the new millennium. In this section we put these examples in a larger perspective, from both a technological and a societal viewpoint.

The technology demonstrated in this chapter shows how it is possible to improve the comfort and quality of life for the child by elimination of the adhesive electrodes and by the elimination of wires. In fact, the elimination of wires goes in steps, the first of which is the decision to transfer signals via radio rather than by wired transmission. In order to make this happen, the amplifiers and filters must move from the remote monitoring area into the body area which introduces the need for energy to power the amplifiers, filters and radio transmitters. This, in turn, introduces the need for local energy, either through new wires, batteries or by wireless energy transmission. Therefore the second step is to eliminate this local energy problem, which is precisely what the PowerBoy system does. Bringing the amplifiers and the filters closer to the body will give an additional advantage, which is not fully exploited yet in the current version of the smart jacket. The advantage will be that all the electric interference picked up by the traditional long leads is strongly reduced. Still, precautions will be needed to prevent the newly introduced power-supply and radiotransmission carriers from inducing new artifacts, notably in the pre-amplifier stages. For 
the time being, some care is thus needed with pulse and amplitude based modulation techniques. On the long term, ultra-low power transmission techniques will take care of this potential problem. Another concern is the question whether the newly introduced highfrequency fields could be harmful for the child. It is advisable to stay on the safe side, which is why the PowerBoy is a separate toy and the child is outside of the field. This is a good solution now. In ten years from now, low power radio and low power photoplethysmography (PPG) sensors could well be available, allowing for full integration of all electronics into the jacket itself. The introduction of textile electrodes is another technological step, which has introduced a new problem. The problem is the signal quality, since the signal is weaker and more sensitive to movement artifacts. An alternative technology would be capacitive electrodes, but these have similar problems. Of course proper placement of the electrodes helps, as shown in the smart jacket design for neonatal monitoring. Multi-modal signal processing will be the way ahead. For example, combining movement sensors, ECG sensors and PPG sensors gives extra information which can be used to automatically distinguish artifacts from genuine heart rate abnormalities.

Taking a societal viewpoint, the smart jacket and power system fit into the ambient intelligence approach. The sensors could become invisible and important monitoring tasks taken over by computers which could become invisible as well. In general, the societal debate about ambient intelligence in health care has hardly begun. In the Netherlands, the report issued by the Rathenau Institute (Schuurman et al., 2007) is one of the examples of the beginning debate. A European perspective can be found in the paper by Duquenoy and Whitehouse (Duquenoy \& Whitehouse, 2006) who explain ambient intelligence as combining developments in information and communication technologies with notions of 'pervasive' and 'ubiquitous' computing, and describing an intelligent environment operating in the background in an invisible and non-intrusive way. Several communities have different views, but doubtlessly problems such as information overload and conflict of governmental and/or commercial interests with private interests will arise. For prematurely born infants, monitoring of vital functions while raising the comfort level is a medical necessity. Gradually it will become possible, however, to transfer the solutions developed for critically ill children towards the larger potential buyer groups (parents of the healthy newborns). These solutions could become modern versions of the old FM audio baby monitors and the present-day baby cams. But is it necessary that parents are reading more and more bodily parameters of their child? Is it wise to collect such data in computers with the possibility that more and more parties get hold of the data? These are not technological questions, but topics for political, social, organizational, economic, legal, regulatory, and ethical debate.

\section{References}

Aarts, E. H. L. \& Encarnação, J. L. (Eds.). (2006). True Visions the Emergence of Ambient Intelligence, Springer-Verlag, Berlin, Heidelberg.

Als, H.; Lawhon, G.; Brown, E.; Gibes, R.; Duffy, H.; Mcanulty, G. B. \& Blickman, J. G. (1986). Individualized behavioral and environmental care for the very low birth weight preterm infant at high risk for bronchopulmonary dysplasia: Neonatal 
intensive care unit and developmental outcome. Pediatrics, Vol. 78, No. 6, 1986, pp. 1123-1132.

Als, H; Gilkerson, L.; Duffy, F. H.; Mcanulty, G. B.; Buehler, D. M.; Vandenberg, K.; Sweet N.; Sell, E.; Parad, R. B.; Ringer, S. A.; Butler, S. C.; Blickman, J. G. \& Jones, K. J. (2003). A three-center, randomized, controlled trial of individualized developmental care for very low birth weight preterm infants: medical, neurodevelopmental, parenting and caregiving Effects. Journal of Developmental and Behavioral Pediatrics, Vol. 24, 2003, pp. 399-408.

Anand, K. J. S. \& Scalzo, F. M. (2000). Can adverse neonatal experiences alter brain development and subsequent behaviour?. Biology of the Neonate, Vol. 77, No. 2, Feb. 2000, pp. 69-82.

Bouwstra, S.; Chen, W.; Feijs, L. M. G. \& Bambang Oetomo, S. (2009). Smart jacket design for neonatal monitoring with wearable sensors, Proceedings of Body Sensor Networks (BSN 2009), pp. 162 - 167, Berkeley, USA, June 2009.

Catrysse, M.; Hermans B. \& Puers, R. (2004). An inductive power system with integrated bidirectional data-transmission. Sensors and Actuators A: Physical, Vol. 115, No. 2-3, 21 September 2004, pp. 221-229.

Chapieski, M. L. \& Evankovitch, K. D. (1997). Behavioral effects of prematurity. Semin. Perinatol., Vol. 21, 1997, pp. 221-239.

Chen, W.; Sonntag, C. L. W.; Boesten, F.; Bambang Oetomo, S. \& Feijs, L. M. G. (2008). A power supply design of body sensor networks for health monitoring of neonates, Proceedings of the Fourth International Conference on Intelligent Sensors, Sensor Networks and Information Processing (ISSNIP 2008), pp.255-260, Sydney, Australia, Dec. 2008.

Chen, W.; Nguyen S. T.; Coops, R.; Bambang Oetomo, S. \& Feijs, L. M. G. (2009a). Wireless transmission design for health monitoring at neonatal intensive care units, submitted to the 2nd international symposium on applied sciences in biomedical and communication technologies (ISABEL 2009), Bratislava, Slovak Republic, Nov. 2009.

Chen, W.; Sonntag, C. L. W.; Boesten, F.; Bambang Oetomo, S. \& Feijs, L. M. G. (2009b). A design of power supply for neonatal monitoring with wearable sensors. Journal of Ambient Intelligence and Smart Environments-Special Issue on Wearable Sensors, Vol.1, No. 2, 2009, pp. 185 - 196, IOS press.

Chen, W.; Ayoola, I. B. I.; Bambang Oetomo, S. \& Feijs, L. M. G. (2010a). Non-invasive blood oxygen saturation monitoring for neonates using reflectance pulse oximeter, submitted to Design, Automation and Test in Europe - Conference and Exhibition 2010 (DATE 2010), Dresden, Germany, March 2010.

Chen, W.; Bambang Oetomo, S. \& Feijs, L. M. G. (2010b). Neonatal monitoring - current practice and future trends. Handbook of Research on Developments in e-Health and Telemedicine: Technological and Social Perspectives, IGI Global, to be published in 2010.

Chen, W.; Dols, S.; Bambang Oetomo, S. \& Feijs, L. M. G. (2010c). Monitoring body temperature of a newborn baby", to be submitted to the Eighth Annual IEEE International Conference on Pervasive Computing and Communications (PerCom 2010), Mannheim, Germany, March 2010. 
Costeloe, K.; Hennessy, E.; Gibson, A. T.; Marlow, N. \& Wilkinson, A. R. (2000). The EPICure study: Outcome to discharge from hospital for infants born at the threshold of viability. Pediatrics, Vol. 106, No. 4, 2000, pp. 659-671.

de Kleine, M. J.; den Ouden, A. L.; Kollée, L. A.; Ilsen, A.; van Wassenaer, A. G.; Brand R. \& Verloove -Vanhorick, S.P. (2007). Lower mortality but higher neonatal morbidity over a decade in very preterm infants. Paediatr Perinat Epidemiol, Vol. 21, No. 1, 2007, pp. 15-25.

Duquenoy, P. \& Whitehouse, D. (2006). A 21st century ethical debate: pursuing perspectives on ambient intelligence. IFIP International Federation for Information Processing, the Information Society: Emerging Landscapes, Vol. 195, 2006, pp 293-314, Springer Boston.

Goldsmith, A. (2005). Wireless Communications, Cambridge University Press.

Hack, M. \& Fanaroff, A. A. (1999). Outcomes of children of extremely low birth weight and gestational age in the 1990's. Early Hum Dev., Vol. 53, 1999, pp. 193-218.

Hayles, P. (2008). Intelligent NiCd battery charger. [Online], Retrieved 2008. Available: http://www.angelfire.com/electronic/hayles/charge1.html.

International Commission on Non-Ionizing Radiation Protection (ICNRP) (1998). Guidelines for limiting exposure to time-varying electric, magnetic, and electromagnetic fields (up to 300 GHz). Health Physics Society, Vol. 74, No. 4, pp. 494-522, April 1998.

Ma, G.; Yan, G. \& He, X. (2007). Power transmission for gastrointestinal microsystems using inductive coupling. Physiol. Meas., Vol. 28, 2007, pp. N9-N18.

Marlow, N.; Hennessy, E. M.; Bracewell, M. A. \& Wolke, D. (2007). Motor and executive function at 6 years of age after extremely preterm birth. Pediatrics, Vol. 120, No. 4, 2007, pp. 793-804.

Murković, I.; Steinberg, M. D. \& Murković, B. (2003). Sensors in neonatal monitoring: Current practice and future trends. Technology and Health Care, Vol. 11, IOS Press, 2003, pp. 399-412.

Paradiso, J. A. \& Starner, T. (2005). Energy scavenging for mobile and wireless electronics. IEEE Pervasive Comput., Vol. 4, No. 1, 2005, pp. 18-27.

Perlman, J. M. (2001). Neurobehavioral deficits in premature graduates of intensive care Potential medical and environmental risks factors. Pediatrics, Vol. 108, No. 6, 2001, pp. 1339-1348.

Perlman, J. M. (2003). The genesis of cognitive and behavioural deficits in premature graduates of intensive care. Minerva Pediatr., Vol. 55, 2003, pp. 89-101.

Polin, R. A. \& Fox, W. W. (Eds.). (1992). Fetal and Neonatal Physiology, W. B. Saunders Company.

Qin, Y.; Wang, X. \& Wang, Z. L. (2008). Microfibre-nanowire hybrid structure for energy scavenging. Nature, Vol. 451, 14 Feb. 2008, pp. 809 - 813.

Schuurman, J.; El-Hadidy, F.; Krom, A. \& Walhout B. (2007). Ambient Intelligence. Toekomst van de zorg of zorg van de toekomst? Rathenau Instituut, Den Haag:

Sonntag, C. L. W.; Lomonova, E. A. \& Duarte, J. L. (2008). Power transfer stabilization of the three-phase contactless energy transfer desktop by means of coil commutation, Proceedings of the 4th IEEE Young Researchers Symposium in Electrical Engineering (YRS 2008), pp. 1-6, Eindhoven, the Netherlands, February 2008. 
Tao, X. M. (Ed.). (2005). Wearable Electronics and Photonics, CRC press, Woodhead Publishing Ltd., England.

Van Langenhove, L. (Ed.). (2007). Smart Textiles for Medicine and Healthcare: Materials, Systems and Applications, CRC press, Woodhead Publishing Ltd., England.

Yang, G. Z. (Ed.). (2006). Body Sensor Networks, Springer-Verlag London Limited. 


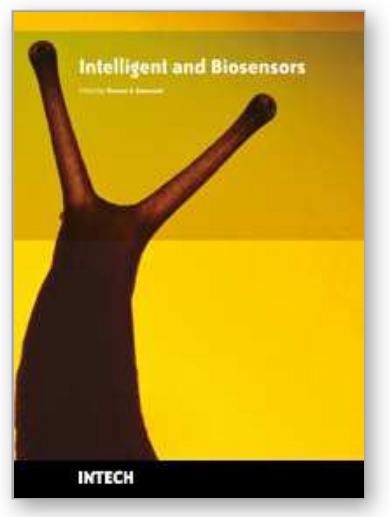

\author{
Intelligent and Biosensors \\ Edited by Vernon S. Somerset
}

ISBN 978-953-7619-58-9

Hard cover, 386 pages

Publisher InTech

Published online 01, January, 2010

Published in print edition January, 2010

The use of intelligent sensors have revolutionized the way in which we gather data from the world around us, how we extract useful information from that data, and the manner in which we use the newly obtained information for various operations and decision making. This book is an attempt to highlight the current research in the field of Intelligent and Biosensors, thereby describing state-of-the-art techniques in the field and emerging new technologies, also showcasing some examples and applications.

\title{
How to reference
}

In order to correctly reference this scholarly work, feel free to copy and paste the following:

Wei Chen, Sibrecht Bouwstra, Sidarto Bambang Oetomo and Loe Feijs (2010). Intelligent Design for Neonatal Monitoring with Wearable Sensors, Intelligent and Biosensors, Vernon S. Somerset (Ed.), ISBN: 978-9537619-58-9, InTech, Available from: http://www.intechopen.com/books/intelligent-and-biosensors/intelligentdesign-for-neonatal-monitoring-with-wearable-sensors

\section{INTECH}

open science | open minds

\author{
InTech Europe \\ University Campus STeP Ri \\ Slavka Krautzeka 83/A \\ 51000 Rijeka, Croatia \\ Phone: +385 (51) 770447 \\ Fax: +385 (51) 686166 \\ www.intechopen.com
}

\author{
InTech China \\ Unit 405, Office Block, Hotel Equatorial Shanghai \\ No.65, Yan An Road (West), Shanghai, 200040, China \\ 中国上海市延安西路65号上海国际贵都大饭店办公楼 405 单元 \\ Phone: +86-21-62489820 \\ Fax: $+86-21-62489821$
}


(C) 2010 The Author(s). Licensee IntechOpen. This chapter is distributed under the terms of the Creative Commons Attribution-NonCommercialShareAlike-3.0 License, which permits use, distribution and reproduction for non-commercial purposes, provided the original is properly cited and derivative works building on this content are distributed under the same license. 